\title{
$\beta$-Ketoacyl acyl carrier protein reductase (FabG) activity of the fatty acid biosynthetic pathway is a determining factor of 3-oxo-homoserine lactone acyl chain lengths
}

\author{
Tung T. Hoang,† Sarah A. Sullivan, $\ddagger$ John K. Cusick $\mathbb{S}$ \\ and Herbert P. Schweizer
}

Department of

Microbiology, Immunology and Pathology, Colorado State University, Fort Collins, CO 80523-1682A, USA
Author for correspondence: Herbert P. Schweizer. Tel: +1 970491 3536. Fax: +1 9704911815. e-mail: herbert.schweizer@colostate.edu

The two acyl-homoserine lactones (AHLs) $\mathbf{N}$-(butyryl)-L-homoserine lactone and $\mathrm{N}$-[3-oxododecanoyl]-L-homoserine lactone (3-oxo- $\mathrm{C}_{12}-\mathrm{HSL}$ ) are required for quorum sensing in Pseudomonas aeruginosa. These AHLs derive their invariant lactone rings from $S$-adenosylmethionine and their variable acyl chains from the cellular acyl-acyl carrier protein (ACP) pool. This reaction is catalysed by specific AHL synthases, which exhibit acyl chain specificity. Culture supernatants of $\boldsymbol{P}$. aeruginosa contain multiple 3-0xo-AHLs that differ in their acyl chain lengths but their physiological role, if any, remains unknown. An in vitro fatty acid-3-oxo-AHL synthesis system was established utilizing purified P. aeruginosa Fab proteins, ACP and the LasI 3-oxo-AHL synthase. In the presence of excess protein, substrates and cofactors, this system produced almost exclusively 3-oxo- $\mathrm{C}_{12}$-HSL. When the $\beta$-ketoacyl-ACP reductase (FabG) catalysed step was made rate-limiting by switching from the preferred NADPH cofactor to NADH, increased levels of short chain 3-oxo-AHLs were produced, presumably because shorter-chain ketoacyl-ACPs accumulated and thus became Lasl substrates. Consistent with these in vitro observations, a fabG(Ts) mutant produced increased amounts of 3-oxo-AHLs in vivo. Thus, in vitro and in vivo evidence indicated that modulation of FabG activity of the fatty acid biosynthetic pathway may determine the acyl chain lengths of these 3-oxoAHLs and that the LasI 3-0xo-AHL synthase is sufficient for their synthesis.

Keywords: Pseudomonas, homoserine lactone, fatty acid synthesis, synthase

\section{INTRODUCTION}

The expression of many extracellular Pseudomonas aeruginosa virulence factors (Passador et al., 1993; Van Delden \& Iglewski, 1998) and other cellular processes, such as biofilm maturation in vitro (Davies et al., 1998) and biofilm formation in the lungs of cystic fibrosis

\footnotetext{
† Present address: Department of Microbiology, University of Hawaii at Manoa, Honolulu, HI 96822, USA.

$\ddagger$ Present address: VWR International, 106 Gray Road, Suite D, Indianapolis, IN 46237, USA.

\Present address: National Jewish Hospital, 1400 Jackson Street, Denver, CO 80206, USA.

Abbreviations: $A C P$, acyl carrier protein; $A H L$, acyl homoserine lactone; $\mathrm{C}_{4}$-HSL, $\mathrm{N}$-(butyryl)-L-homoserine lactone; Fab, fatty acid biosynthesis; HSL, homoserine lactone; SAM, S-adenosyl methionine.
}

patients (Singh et al., 2000) are regulated in a celldensity-dependent manner by a process called cell-tocell communication or quorum sensing. Cell-to-cell communication in $P$. aeruginosa involves the two acyl homoserine lactones (AHLs) $N$-(butyryl)-L-homoserine lactone $\left(\mathrm{C}_{4}\right.$-HSL) and $\mathrm{N}$-[3-oxododecanoyl]-L-HSL (3oxo- $\mathrm{C}_{12}$-HSL). Although these two AHLs seem to be the main players involved in quorum sensing, $P$. aeruginosa produces other AHLs which differ by their acyl chain lengths but their physiological roles, if any, remain unclear. A quinolone signal (Pesci et al., 1999) and perhaps cyclic peptides (Holden et al., 1999) also seem to participate in some of these regulatory networks.

Several previous studies revealed that bacterial AHLs derive their invariant homoserine lactone rings from $S$ adenosyl methionine (SAM) and their variable acyl 

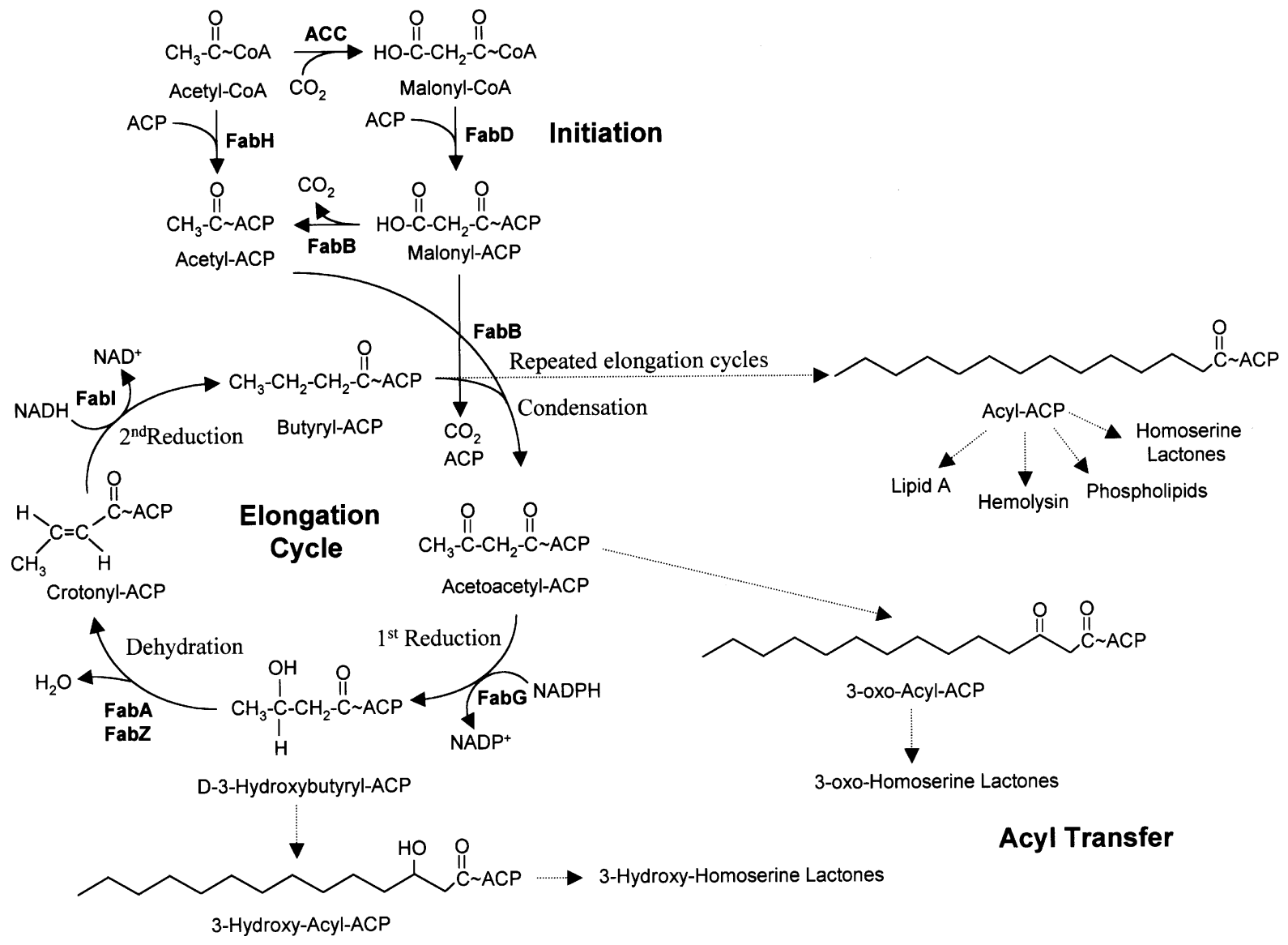

Acyl Transfer

Fig. 1. Fatty acid biosynthesis in $P$. aeruginosa, and acyl-ACPs as acyl donors in cellular metabolism and AHL synthesis. There are several potential pathways to generate acetoacetyl-ACP and initiate fatty acid synthesis (Cronan \& Rock, 1996) but not all are shown for the sake of clarity. In the reaction shown, which explains the in vitro system established in this study, malonyl-ACP is decarboxylated to acetyl-ACP by FabB, which then condenses these two molecules to acetoacetylACP to initiate the cycle (Cronan \& Rock, 1996). The malonyl-ACP is derived from malonyl-CoA by malonyl-CoA:ACP acyltransferase (FabD). Subsequent cycles are initiated by condensation of malonyl-ACP with acyl-ACP, catalysed by FabB ( $\beta$-ketoacyl ACP synthase I). The $\beta$-ketoacyl-ACP from the FabB reaction is reduced to a $\beta$-hydroxyacyl-ACP by FabG, a NADPH-dependent $\beta$-ketoacyl-ACP reductase. The subsequent dehydration step is catalysed by either FabA or FabZ, depending on the lengths of the acyl groups in the $\beta$-hydroxyacyl-ACP substrates. The final step involves reduction of the dehydratase product to an acyl-ACP via Fabl, a NADH-dependent enoyl-ACP reductase. Subsequent cycles are initiated by a FabB-catalysed condensation of malonyl-ACP with acyl-ACP. For synthesis of 3-oxo- $\mathrm{C}_{12}-\mathrm{HSL}$, Lasl utilizes the 3-oxododecanoyl-ACP from the Fab pathway. Similarly, Rhll uses crotonyl-ACP for synthesis of $\mathrm{C}_{4}-\mathrm{HSL}$. Enzymes involved in 3$\mathrm{OH}-\mathrm{AHL}$ synthesis probably use D-3-hydroxy-ketoacyl-ACP substrates from the Fab cycle. Other biosynthetic pathways, including the phospholipid, lipopolysaccharide, haemolysin and other pathways, also use acyl-ACP intermediates. Other abbreviations: ACC, acetyl-CoA carboxylase; ACP, acyl carrier protein; FabH, $\beta$-ketoacyl ACP synthase III.

chains from the cellular acyl-ACP (acyl carrier protein) pool (Hoang \& Schweizer, 1999; Moré et al., 1996; Parsek et al., 1999; Val \& Cronan, 1998) (Fig. 1). Acyl chain specificity resides in critical amino acid residues within the AHL synthase sequences (Watson et al., 2002). The AHL synthases (LasI for 3-oxo- $\mathrm{C}_{12}-\mathrm{HSL}$ and RhlI for $\mathrm{C}_{4}$-HSL) are sufficient for catalysis of the acyl transfer and lactonization reactions (Moré et al., 1996; Parsek et al., 1999; Hoang \& Schweizer, 1999; Hoang et al., 1999). P. aeruginosa culture supernatants contain 3-oxo-AHLs with various acyl chain lengths but their metabolic origins have not been elucidated. In this study, we attempted to elucidate the molecular basis for the synthesis of these 3-oxo-AHLs. Since LasI competes with NADPH-dependent $\beta$-ketoacyl-ACP reductase, FabG, for the 3-oxo-acyl-ACP precursors for synthesis of these 3-oxo-AHLs (Fig. 1), we reasoned that FabG activity may be a modulating factor determining acyl chain lengths in 3-oxo-AHLs. Because most Fab (fatty acid biosynthesis) enzymes, including FabG, are essential, conventional mutant analysis cannot be used to address their roles in cellular metabolism. To circumvent these problems, a complete in vitro Fab system using purified Escherichia coli Fab proteins and ACP was previously described and was shown to produce the types and distribution of acyl-ACP species found in vivo (Heath \& Rock, 1996a, b). Since the E. coli and P. aeruginosa Fab systems are quite similar, we reasoned 
that an in vitro Fab-3-oxo-AHL synthesis system could be used to explore FabG activity as a factor determining acyl chain lengths of 3-oxo-AHLs. To this end, we purified the $P$. aeruginosa $\mathrm{Fab}$ proteins as hexahistidine $\left(\mathrm{H}_{6}\right)$ fusion proteins and developed an in vitro Fab-AHL synthesis system by coupling them to purified LasI. Some of the observations made with the in vitro system were supported by preliminary in vivo data obtained with a conditional, temperature-sensitive $f a b G(T s)$ mutant.

\section{METHODS}

Strains and growth media. Escherichia coli strains used in this study were DH5 $\alpha$ (Liss, 1987), BL21(DE3) (Novagen), SA1503(DE3) (Hoang et al., 1999) and the 3-oxo-C 12 -HSL reporter strain MG4/pKDT17 (lasR ${ }^{+}$lasB-lacZ) (Schaefer et al., 2000). The wild-type $P$. aeruginosa strain PAO1 was previously described (Watson \& Holloway, 1978). P. aeruginosa strain 4 is a clinical wound isolate from the Glaxo SmithKline collection and is similar to PAO1 in terms of extracellular protein profiles, exoenzyme $S$ production and nucleotide sequences. The $f a b G($ Ts $)$ mutant ts- 67 was derived from strain 4 by Dr J. Huang (Collegeville, PA, USA) at Glaxo SmithKline and strain ts-67R1 is a revertant of strain ts-67. The Agrobacterium tumefaciens strains NTL4/pZLR4 (containing traR and traG::lacZ) and NT1/pTiC58 $\triangle a c c R$ were from S. Farrand (University of Illinois, Urbana, USA). The Erwinia carotovora strain EC14 was previously described (Schweizer, 1994). Unless otherwise indicated, bacterial strains were grown in LB medium (Difco), which for plasmid maintenance in E. coli was supplemented with $100 \mu \mathrm{g}$ ampicillin $\mathrm{ml}^{-1}$ and/or $25 \mu \mathrm{g}$ chloramphenicol $\mathrm{ml}^{-1}$.

Construction of expression vectors and affinity purification of proteins. The coding sequences for the individual enzymes were PCR amplified from PAO1 genomic DNA utilizing Taq polymerase and previously described conditions (Hoang \& Schweizer, 1999; Hoang et al., 1998). The general strategy involved the use of a forward primer that incorporated an NdeI restriction site at the start codon of the respective gene and a reverse primer that incorporated a BamHI restriction site after the stop codon of the same gene (Table 1$)$. The gel- purified (QIAquick gel extraction kit; Qiagen) PCR fragments were digested with $\mathrm{NdeI} / \mathrm{Bam} \mathrm{HI}$ and then ligated between the same sites of pET-15b (Novagen). Since fabG contained a $B a m \mathrm{HI}$ site, the reverse primer incorporated a $B g l \mathrm{II}$ site, which allowed subcloning into the BamHI site of pET-15b. Standard molecular biological techniques were used (Sambrook \& Russell, 2001). Subcloning into pET-15b yielded the expression vectors pPS837 (FabB), pPS980 (FabG), pPS998 (FabH) and pPS937 (FabZ). For FabA, the PCR fragment was first cloned into the TA cloning vector pGEM-T (Promega) to yield pPS847. An NdeI-BamHI fragment derived from this plasmid was then subcloned between the same sites of pET-15b (Novagen) to yield the FabA expression vector pPS848. For expression of the resulting proteins with $\mathrm{NH}_{2}$-terminal hexahistidine $\left(\mathrm{H}_{6}\right)$ tags, the plasmids were transformed into BL21(DE3) (Novagen). Screening of $\mathrm{H}_{6}$-Fab protein-expressing transformants, cell lysis and purification of the soluble fusion proteins on $\mathrm{Ni}^{2+}$ agarose affinity columns (Qiagen) was performed as previously described (Hoang et al., 1999), except for FabD. Since FabD eluted from the columns with $40 \mathrm{mM}$ imidazole, washing of the column was done with 30 bed vols buffer with $20 \mathrm{mM}$ imidazole.

ACP was purified via an intein chitin-binding domain fusion protein as previously described (Kutchma et al., 1999), except that it was coexpressed with acyl-ACP synthase (AcpS) to maximize holo-ACP formation. To this end, the AcpS expressing pPS1118 was constructed by subcloning the acpS gene from E. coli on a $470 \mathrm{bp}$ AseI-HindIII fragment from pDPJ (Lambalot \& Walsh, 1995) between the same sites of pACYC184 (Chang \& Cohen, 1978). For coexpression of ACP and AcpS, the expression strain was grown in LB + ampicillin + chloramphenicol medium to maintain the acpP-and acpScontaining plasmids. $\mathrm{H}_{6}$-LasI was purified using a published procedure (Hoang et al., 1999).

Protein concentrations were determined using the Bradford dye-binding assay (Bio-Rad) and BSA as the standard. Proteins were analysed by electrophoresis on $0 \cdot 1 \%$ SDS- $10 \%$ polyacrylamide gels (SDS-PAGE) (Makowski \& Ramsby, 1993) and visualized by staining with Coomassie Brilliant Blue R250 (Chen et al., 1993).

Complementation assays. The coding sequences for the $\mathrm{H}_{6}$ tagged FabA, FabB and FabD proteins were subcloned into the

\section{Table 1. List of PCR primers}

The forward primers incorporated an NdeI restriction site (underlined) at the start codon of the respective genes. In most cases, the reverse primers incorporated a BamHI restriction site (underlined) after the stop codon with the exception of FabG (reverse), which incorporated a BglII site after the fabG stop codon.

\begin{tabular}{|ll|}
\hline Name & Sequence $\left(\mathbf{5}^{\prime} \mathbf{- 3}^{\prime}\right)$ \\
\hline FabA (forward) & TCATATGACCAAACAACACGCCTTCAC \\
FabA (reverse) & GGATCCCCCTTAGAAGCTGTCAGTGGAG \\
FabB (forward) & TCCATATGCGTCGCGTCGTTATCACCGGTC \\
FabB (reverse) & ATGGATCCAATCAACCCTGCCAGCGCTTGAGGA \\
FabG (forward) & TGACATATGCCGCGCGCCGCCGTGGTCT \\
FabG (reverse) & GACAGATCTTATGACAGACCCGAGAAAGGTAAC \\
FabH (forward) & TGACATATGCCGCGCGCCGCCGTGGTCT \\
FabH (reverse) & GTGGATCCCTCTTCAGTCCATTGTCGG \\
FabZ (forward) & CCTCATATGATGGACATCAACGAGATTCG \\
FabZ (reverse) & GAGGATCCATCAAACTCATAGTTTGCGT \\
\hline
\end{tabular}


broad-host-range vector pUCP21T (Schweizer et al., 1996) on BamHI-XbaI fragments. Subcloning between the BamHI and $X b a \mathrm{I}$ sites of pUCP21T placed the $\mathrm{H}_{6}$-Fab coding sequences in the correct transcriptional orientation with respect to the lac promoter contained on this cloning vector and yielded pPS1013 $\left(\mathrm{H}_{6}-\mathrm{FabA}\right)$, pPS1025 $\left(\mathrm{H}_{6}-\mathrm{FabB}\right)$ and pPS1019 $\left(\mathrm{H}_{6}{ }^{-}\right.$ FabD). To test for expression of functional $\mathrm{H}_{6}-\mathrm{FabA}$ and $\mathrm{H}_{6}-$ FabB proteins, pPS1013 and pPS1025 were transformed (Hoang et al., 1998) into strain PAO191 (fabA) and PAO192 $(f a b B)$ (Hoang \& Schweizer, 1997), respectively. Since FabA and $\mathrm{FabB}$ are required for unsaturated fatty acid synthesis, PAO191 and PAO192 will not grow at $42{ }^{\circ} \mathrm{C}$ unless supplemented with oleic acid or complemented with either a FabA- or FabB-expressing plasmid. Complementation was therefore scored as the ability to grow at $42^{\circ} \mathrm{C}$ on RB medium without oleate supplementation (Hoang \& Schweizer, 1997). To test for expression of a functional $\mathrm{H}_{6}-\mathrm{FabD}$ protein, pPS1019 was transformed into the $\mathrm{fabD}$ (Ts) mutant PAO204 (Kutchma et al., 1999). Successful complementation was scored as the ability of the transformants to grow on LB plates at $42^{\circ} \mathrm{C}$. In all instances, strains were transformed with pUCP21T as a negative control.

Reconstitution of the Fab-AHL pathway and extraction of 3oxo-acyl-HSLs. Complete reactions (total volume $500 \mu \mathrm{l}$ ) contained buffer $[10 \mathrm{mM}$ Tris $/ \mathrm{HCl}(\mathrm{pH} 7 \cdot 4), 330 \mathrm{mM} \mathrm{NaCl}$, $15 \%$, w/v, glycerol, $0.7 \mathrm{mM}$ DTT, $2 \mathrm{mM}$ EDTA, $25 \mathrm{mM}$ $\mathrm{MgSO}_{4}, 0 \cdot 1 \mathrm{mM} \mathrm{FeSO}_{4}$ ] (Moré et al., 1996), $2 \mu \mathrm{g} \mathrm{ACP}, 1 \mu \mathrm{g}$ each FabA, FabB, FabD, FabH, FabI and FabZ, 0.5 $\mu$ g FabG, $5 \mu \mathrm{g}$ LasI, $0.25 \mathrm{mM}$ SAM, $0.08 \mathrm{mM}$ acetylCoA, $0.8 \mathrm{mM}$ malonyl-CoA and $0.6 \mathrm{mM}$ each NADH and NADPH (substrates and cofactors were obtained from Sigma). Reactions were incubated at $37^{\circ} \mathrm{C}$ for $1 \mathrm{~h}$ and extracted three times with $250 \mu \mathrm{l}$ ethyl acetate. Extracted AHLs were dried by rotary vacuum evaporation and resuspended in $20 \mu \mathrm{l}$ acetonitrile. For detection of fractions containing AHLs, 5-10 $\mu$ l each fraction was spotted on $\mathrm{C}_{18}$ reverse-phase TLC plate (Whatman) and the plates were dried at $37^{\circ} \mathrm{C}$ for $15 \mathrm{~min}$ before being overlaid with the detection strain. For TLC analysis of AHL fractions, the plates were developed in $60 \%$ methanol in water $(\mathrm{v} / \mathrm{v})$ and then dried for $20 \mathrm{~min}$ at $37^{\circ} \mathrm{C}$ prior to being overlaid with the detection strain.

Detection, identification and quantification of AHLs. A. tumefaciens reporter strain NTL4/pZLR4 was grown at $30{ }^{\circ} \mathrm{C}$ for $48 \mathrm{~h}$ in $\mathrm{M} 9$ medium (Miller, 1992) with $1 \mathrm{mM} \mathrm{MgSO}_{4}$, $0 \cdot 1 \mathrm{mM} \mathrm{CaCl}_{2}, 0 \cdot 6 \%$ glucose and $30 \mu$ gentamicin $\mathrm{ml}^{-1}$ (Shaw et al., 1997). Cells were harvested and resuspended in warm $\left(\sim 45^{\circ} \mathrm{C}\right.$ ) fresh $\mathrm{M} 9$ medium with $0.4 \%$ agar, $1 \mathrm{mM} \mathrm{MgSO}{ }_{4}$, $0 \cdot 1 \mathrm{mM} \mathrm{CaCl}, 0 \cdot 6 \%$ glucose and $40 \mu \mathrm{g} \mathrm{X}-\mathrm{Gal} \mathrm{ml}^{-1}$. This suspension was used immediately to overlay the TLC plates. The presence of AHLs was usually evident by the appearance of blue spots after incubation at room temperature for 36$48 \mathrm{~h}$. Synthetic 3-oxo- $\mathrm{C}_{12}$-HSL, and bacterial-derived 3-oxo$\mathrm{C}_{8}$-HSL and 3-oxo- $\mathrm{C}_{6}$-HSL were included as standards. The latter two were extracted from $10 \mathrm{ml}$ stationary-phase clarified culture supernatants of A. tumefaciens strain NT1/ pTiC58 $\triangle a c c R$ or Erw. carotovora strain EC14, respectively, using a previously described method (Shaw et al., 1997). The concentrations of 3-oxo- $\mathrm{C}_{12}$-HSL were estimated utilizing the Esc. coli reporter strain MG4/pKDT17 (las $R^{+}$lasB-lacZ) as previously described (Schaefer et al., 2000) and by using a dilution series of synthetic 3 -oxo- $\mathrm{C}_{12}$-HSL to establish a standard curve. For determination of HSL levels in the supernatants of the $f a b G(\mathrm{Ts})$ mutant ts-67, its parental strain 4 and the ts-67R1 revertant of strain ts-67, the strains were grown in LB medium until the cultures reached an optical density of $\sim 1.6(600 \mathrm{~nm})$. The $\mathrm{pH}$ in the cultures was monitored to avoid excess alkalinization of the medium since AHLs are very unstable at alkaline $\mathrm{pH}$ values (Schaefer $e t$ al., 2000). Aliquots ( $1 \mathrm{ml})$ were harvested by centrifugation. The supernatants were extracted three times with $1 \mathrm{ml}$ acidified ethyl acetate (ethyl acetate containing $0 \cdot 1 \mathrm{ml}$ glacial acetic acid per litre), dried and suspended in $200 \mu \mathrm{l}$ acidified ethyl acetate. For detection of fractions containing 3-oxo-HSLs, $10 \mu \mathrm{l}$ each fraction was spotted on $\mathrm{C}_{18}$ reverse-phase TLC plate. The plates were processed as described above and then overlaid with the A.tumefaciens detection strain.

\section{RESULTS AND DISCUSSION}

\section{Purification and in vivo activity of Fab proteins}

Our initial goal was to set up a complete in vitro FabAHL synthesis system using only $P$. aeruginosa proteins by coupling purified Fab enzymes to LasI 3-oxo-AHL synthase. Since we previously described the purification and activity of ACP (Kutchma et al., 1999), FabD (Kutchma et al., 1999), FabI (Hoang \& Schweizer, 1999) and LasI (Hoang et al., 1999), we still needed to purify FabA, FabB, FabG, FabH and FabZ, assuming that all of these proteins are needed to synthesize acyl-ACPs from acetyl-CoA and malonyl-CoA (Fig. 1). Using nondenaturing conditions and metal chelation affinity chromatography, all Fab proteins were purified to near homogeneity after overexpression in E. coli (Fig. 2). When expressed in vivo from the lac promoter, the genes encoding the $\mathrm{H}_{6}$-tagged FabA, FabB and FabD proteins complemented the corresponding $P$. aeruginosa mutations, indicating that the constructs expressed enzymically active $\mathrm{H}_{6}-\mathrm{Fab}$ proteins. We previously showed that

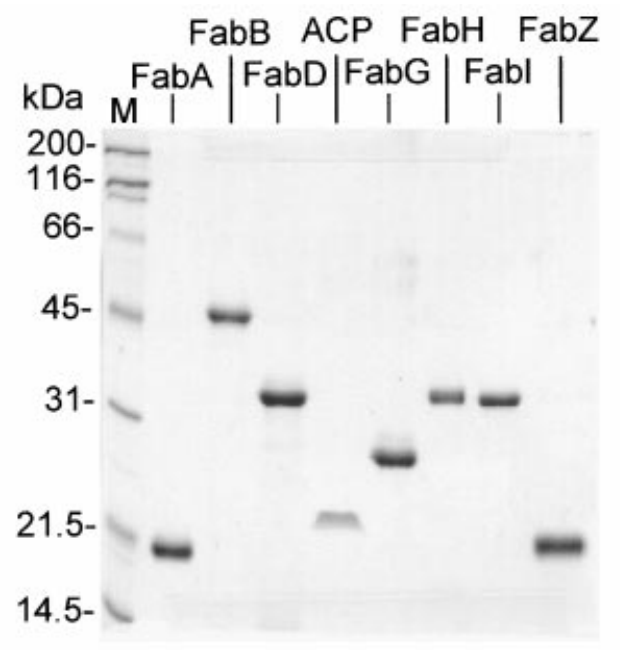

Fig. 2. Gel electrophoretic analysis of purified proteins. Samples of purified ACP and the various Fab proteins were analysed by electrophoresis on a $0 \cdot 1 \%$ SDS-13\% PAGE. The gel was stained with Coomassie blue. All proteins, except $A C P$, were purified with $\mathrm{NH}_{2}$-terminal $\mathrm{H}_{6}$-tag containing extensions. $\mathrm{ACP}$ was purified in its native form via an ACP-intein chitin-binding domain fusion protein. The sizes of protein markers (M) from Bio-Rad are indicated in $\mathrm{kDa}$ and were (top to bottom): myosin, $\beta$-galactosidase, BSA, ovalbumin, carbonic anhydrase, trypsin inhibitor and lysozyme. 


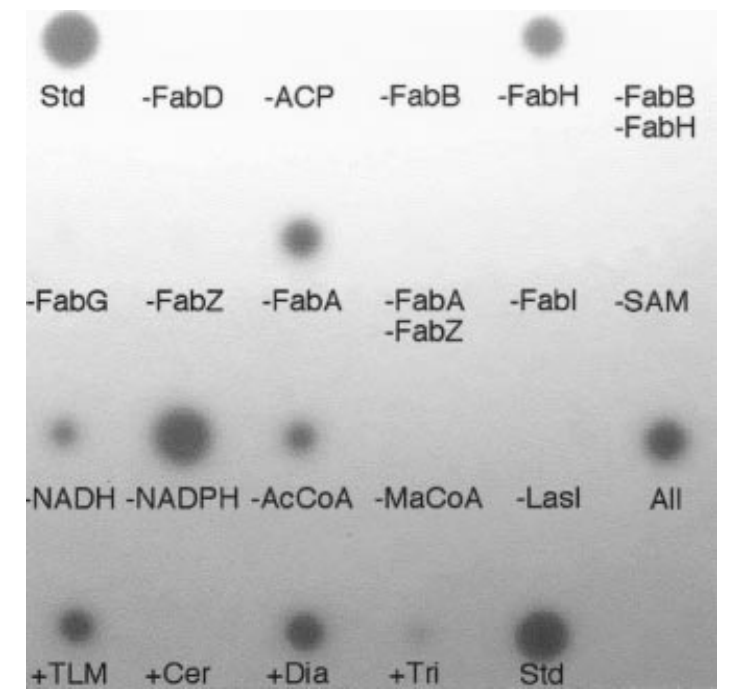

Fig. 3. Enzymic synthesis of 3-oxo-AHLs in a reconstituted enzyme system. Ethyl acetate extracts of reactions were analysed for the presence of 3-oxo-AHLs by spotting samples on a $\mathrm{C}_{18}$-reverse-phase TLC plate and overlaying it with the $A$. tumefaciens NTL4(pZLR4) detection strain in the presence of XGal. Spots indicate the presence of 3-oxo-acyl-HSLs and a 3-oxo$\mathrm{C}_{12}$-HSL standard (Std). The complete reaction (All) contained ACP, FabA, FabB, FabD, FabG, FabH, Fabl, FabZ, Lasl, SAM, acetyl-CoA, malonyl-CoA, and NADH and NADPH. The other reactions lacked the indicated enzymes, substrates or co-factors. Some reactions contained $50 \mu \mathrm{M}$ of the Fab inhibitors thiolactomycin (TLM), cerulenin (Cer), diazaborine (Dia) or triclosan (Tri). Enzyme abbreviations are explained in Fig. 1. Other abbreviations: AcCoA, acetyl-CoA; MaCoA, malonyl-CoA.

expressed $\mathrm{H}_{6}$-FabI complemented an E. coli fabI(Ts) mutant and was enzymically active (Hoang \& Schweizer, 1999). Complementation experiments were not possible for FabH and FabZ since no mutants were available.

\section{Establishment of an in vitro Fab-3-oxo-AHL synthesis system}

The Fab-3-oxo-AHL pathway was reconstituted in vitro and biologically active 3-oxo-AHLs were detected using an A. tumefaciens indicator strain (Fig. 3). The results showed that the minimal Fab-3-oxo-AHL biosynthetic pathway consists of ACP, FabB, FabD, FabG, FabI, FabZ and LasI. Essential metabolites included malonylCoA and SAM. Lesser amounts of 3-oxo-AHLs were produced when acetyl-CoA and NADH were omitted. While our experiments confirmed the previously established importance of some components of the Fab system in AHL synthesis, i.e. the dependency on ACP, metabolites and cofactors (Moré et al., 1996; Parsek et al., 1999; Val \& Cronan, 1998), the minimal pathway was to date unknown and could not have been determined without establishing the experimental system described in this study. The in vitro system also allowed an assessment of the relative contribution of the seemingly redundant components of the Fab system.

Synthases. Since FabB is the major condensing enzyme, it was essential for AHL formation from malonyl-CoA. In contrast, FabH was not required presumably since FabB can decarboxylate malonyl-ACP to acetyl-ACP and then condenses these two molecules to initiate the cycle without FabH (Fig. 1), as has been suggested for E. coli FabB (Cronan \& Rock, 1996). This would also explain the formation of 3-oxo-AHLs in the reactions containing no acetyl-CoA.

Dehydratases. Of the two dehydratases, only FabZ was essential for AHL formation but not FabA. This is probably due to the fact that FabZ is mostly required in the initial cycles since its $E$. coli counterpart has greatest affinity for $\mathrm{C}_{4}-\mathrm{C}_{8} \beta$-hydroxyacyl-ACP intermediates, but can use substrates with longer acyl chains (Heath \& Rock, 1996a). In contrast, E. coli FabA acts preferably on $\mathrm{C}_{10}-\mathrm{C}_{14} \beta$-hydroxyacyl-ACP intermediates.

Reductants. Exclusion of NADH led to detectable AHL production but at much reduced levels. Since NADH is the reductant preferred by FabI (Hoang \& Schweizer, 1999), this result indicates that FabI can utilize NADPH but that this step becomes rate-limiting in the absence of NADH.

When the known Fab inhibitors cerulenin, triclosan, diazoborine and thiolactomycin were added to the reaction mixture, only cerulenin and triclosan efficiently inhibited 3-oxo-AHL formation at the concentration tested $(50 \mu \mathrm{M})$. For unknown reasons, at the same concentrations, thiolactomycin and diazoborine had little effect but from other experiments we suspected that these two antimicrobials, which are not available commercially, had lost much of their activities during storage (data not shown).

\section{Nature of AHL molecules synthesized in vitro}

TLC analysis (Fig. 4) was used to identify AHL species contained in representative positive reactions shown in Fig. 3. The analysis showed that reactions containing all essential components of the Fab-3-oxo-AHL synthesis system almost exclusively yielded 3 -oxo- $\mathrm{C}_{12}$-HSL, and only minute amounts of shorter chain 3-oxo-AHLs were discernible. Conversely, in the absence of NADPH but presence of NADH, LasI synthesized hardly any 3-oxo$\mathrm{C}_{12}$-HSL but larger amounts of 3-oxo- $\mathrm{C}_{10}$-HSL and 3oxo- $\mathrm{C}_{8}$-HSL, and lesser amounts of 3-oxo- $\mathrm{C}_{6}$-HSL (lane labelled -NADPH). Since 3-oxo- $\mathrm{C}_{8}-\mathrm{HSL}$ is the cognate A. tumefaciens AHL, its spot size is not indicative of a higher quantity of 3-oxo- $\mathrm{C}_{8}$-HSL relative to the other 3oxo-AHLs, but rather indicates a better response to its native AHL. According to the pathway model (Fig. 1), LasI and FabG compete for 3-oxo-acyl-ACP substrates from the Fab system. Although FabG can utilize NADH, NADPH is its preferred cofactor and in its absence the FabG-catalysed reduction step becomes rate limiting, leading to accumulation of shorter chain 3-oxo-acyl- 


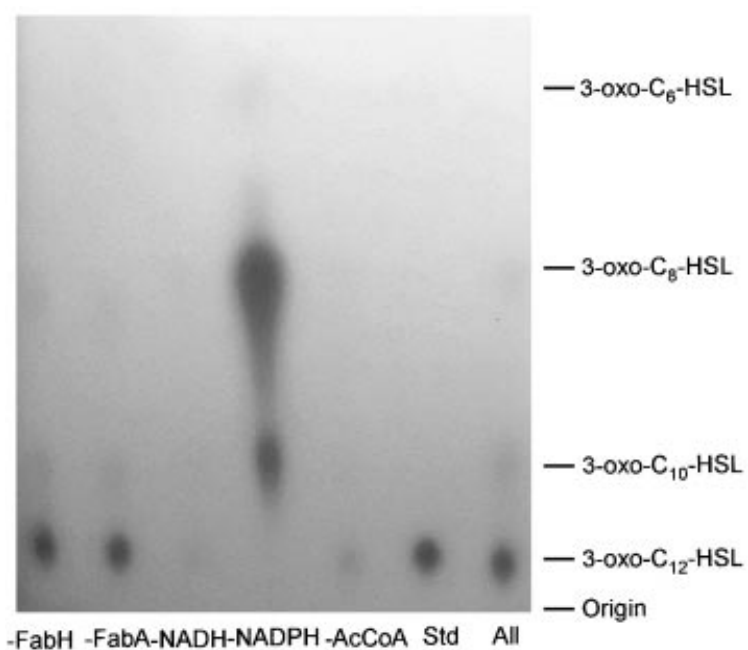

Fig. 4. Identification of AHLs produced in in vitro synthesis reactions. Extracted and concentrated products from selected reactions shown in Fig. 3 were spotted onto a $\mathrm{C}_{18}$-reverse phase TLC plate. The plate was developed with $60 \%(\mathrm{v} / \mathrm{v})$ methanol in water and overlaid with the $A$. tumefaciens NTL4(pZLR4) detection strain in the presence of $\mathrm{X}$-Gal. All, complete reaction mixtures contained ACP, FabA, FabB, FabD, FabG, FabH, Fabl, FabZ, Lasl, SAM, acetyl-CoA, malonyl-CoA, and NADH and $\mathrm{NADPH}$; other reactions lacked the indicated enzymes, substrates or co-factors. The relative mobility of known 3-oxoacyl-HSLs, analysed on the same TLC plate but in a portion that is not shown, and the sample origin are marked on the right.

ACPs. This now enables LasI to compete for the shorterchain 3-oxo-acyl-ACP substrates and use them for synthesis of the corresponding shorter chain 3-oxoAHLs. These results also proved that LasI alone is sufficient for synthesis of the shorter chain 3-oxo-AHLs found in $P$. aeruginosa culture supernatants.

\section{A fabG(Ts) mutant is altered in 3-oxo-AHL production}

A $f a b G(T s)$ mutant was used to obtain preliminary in vivo experimental evidence for some of the in vitro observations. To examine whether altered FabG activity influenced 3-oxo-AHL production in vivo, AHL formation was analysed in a $f a b G(T s)$ mutant grown in $\mathrm{LB}$ medium at permissive temperature $\left(30^{\circ} \mathrm{C}\right)$ and $37^{\circ} \mathrm{C}$, a temperature that is close to non-permissive $\left(38^{\circ} \mathrm{C}\right.$ or higher). The $f a b G(\mathrm{Ts})$ mutant produced elevated levels of all 3-oxo-AHLs at both temperatures, most notably 3oxo- $\mathrm{C}_{6}$ - $\mathrm{HSL}$ which under these experimental conditions was undetectable in supernatants obtained from wildtype and revertant strains, respectively (Fig. 5). Whereas the parental wild-type and the revertant strain produced levels of 3-oxo- $\mathrm{C}_{12}$-HSL that remained nearly constant over the temperature range examined, the $f a b G(T s)$ strain produced elevated levels of this 3-oxo-AHL, which increased with increasing temperatures (Table 2). These increasing 3-oxo- $\mathrm{C}_{12}$-HSL levels were paralleled with a slight decrease in growth rates of the $f a b G(T s)$ mutant as the temperature increased. The doubling

\section{WT TS RE WT TS RE $\quad \mathrm{C}_{6} \quad \mathrm{C}_{8} \mathrm{C}_{12}$}

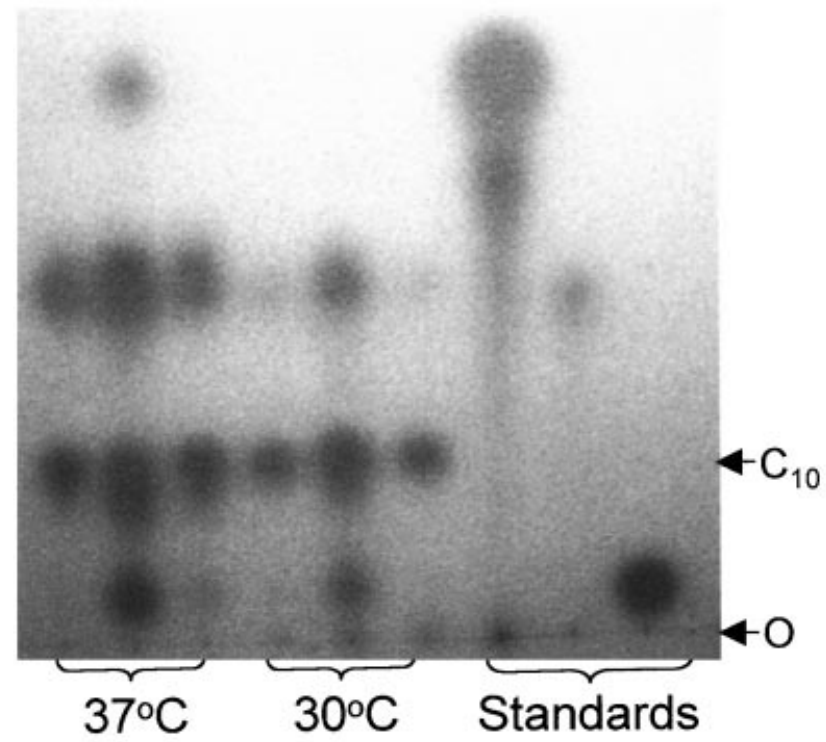

Fig. 5. Identification of 3-oxo-AHLs produced by a fabG(Ts) strain, its parent and a revertant. AHLs were extracted from cells grown at the indicated temperatures and samples of the concentrated reaction products were spotted onto a $\mathrm{C}_{18}$-reverse phase TLC plate. The plate was developed with $60 \%(\mathrm{v} / \mathrm{v})$ methanol in water and overlaid with the $A$. tumefaciens NTL4(pZLR4) detection strain in the presence of X-Gal. Samples analysed were from wild-type strain 4 (WT), its fabG(Ts) derivative (TS) and a revertant that contained a restored wildtype fabG sequence (RE). Standards included 3-oxo- $\mathrm{C}_{6}-\mathrm{HSL}\left(\mathrm{C}_{6}\right)$, 3-oxo- $\mathrm{C}_{8}-\mathrm{HSL}\left(\mathrm{C}_{8}\right)$ and 3-oxo- $\mathrm{C}_{12}-\mathrm{HSL}\left(\mathrm{C}_{12}\right)$. The relative mobility of 3-oxo- $\mathrm{C}_{10}-\mathrm{HSL}\left(\mathrm{C}_{10}\right)$, for which no standard was available, and the origin (O) are marked on the right.

times at $37^{\circ} \mathrm{C}$ were $24 \mathrm{~min}$ for wild-type and revertant, and $36 \mathrm{~min}$ for the $f a b G(\mathrm{Ts})$ mutant. Similar observations to those presented in Fig. 5 and Table 2 were made when AHLs were extracted from cultures grown to lesser cell densities (data not shown). The most plausible explanation for these observations is that even at permissive temperatures the $f a b G(T s)$ strain produces a FabG protein whose reductase activity is decreased when compared to wild-type or revertant FabG. Decreased FabG activity would lead to an increase in the intracellular 3-oxo-acyl-ACP pools, enabling LasI to compete for these substrates, ultimately resulting in increased 3-oxo-acyl-HSL levels.

\section{Conclusions}

The 3-oxo-AHLs normally found in $P$. aeruginosa culture supernatants contain acyl chains of $6-12$ carbons (Shaw et al., 1997) and the relative abundances of different 3-oxo-AHLs change during growth. The results obtained with our in vitro system gave the first clues that modulation of FabG activity by substrate and/or cofactor availability may at least partially explain these observations. In the presence of LasI, this AHL synthase 
Table 2. Estimation of 3-oxo- $\mathrm{C}_{12}-\mathrm{HSL}$ production by a fabG(Ts) mutant, its parent and a revertant

The strains were grown in $\mathrm{LB}$ medium to $\mathrm{OD}_{600} \sim 1.6$ at the indicated temperatures. One millilitre aliquots were harvested by centrifugation. The supernatants were extracted three times with $1 \mathrm{ml}$ acidified ethyl acetate, dried and suspended in $200 \mu \mathrm{l}$ acidified ethyl acetate. The concentrations of 3-oxo- $\mathrm{C}_{12}$-HSL were estimated using the E. coli reporter strain MG4/pKDT17 (lasR ${ }^{+}$lasB-lacZ) and a dilution series of synthetic 3-oxo- $\mathrm{C}_{12}$-HSL to establish a standard curve. The values shown are the means \pm standard deviations of triplicate measurements.

\begin{tabular}{|lccc|}
\hline Strain & \multicolumn{3}{c|}{ Extracellular 3-oxo-C 12 -HSL (nM) } \\
\cline { 2 - 4 } & $\mathbf{2 2}{ }^{\circ} \mathrm{C}$ & $\mathbf{3 0}{ }^{\circ} \mathrm{C}$ & $\mathbf{3 7}{ }^{\circ} \mathrm{C}$ \\
\hline 4 (wild-type) & $48 \cdot 1 \pm 8 \cdot 8$ & $39 \cdot 3 \pm 15 \cdot 0$ & $42 \cdot 6 \pm 15 \cdot 4$ \\
ts-67 [fabG(Ts)] & $252 \pm 88 \cdot 7$ & $301 \pm 79 \cdot 8$ & $487 \pm 133$ \\
ts-67R1 (revertant to wild-type) & $55 \cdot 6 \pm 10 \cdot 7$ & $47 \cdot 1 \pm 25 \cdot 4$ & $26 \cdot 4 \pm 7 \cdot 39$ \\
\hline
\end{tabular}

and FabG compete for 3-oxo-acyl-ACP substrates from the fatty acid biosynthetic pathway. When FabG activity is high, turnover of the short chain 3-oxo-acyl-ACP substrates is rapid and LasI cannot compete for them, presumably because its affinity for them is lower than that of FabG. Once the acyl chain length reaches 12 carbons, LasI efficiently competes for the 3 -oxo- $\mathrm{C}_{12}{ }^{-}$ $\mathrm{ACP}$, resulting in synthesis of 3-oxo- $\mathrm{C}_{12}-\mathrm{HSL}$. When the FabG catalysed step becomes rate limiting, as mimicked in our experimental system by switching cofactors from the preferred NADPH to NADH, accumulation of shorter chain 3-oxo-acyl-ACPs results. This enables LasI to compete for these shorter-chain 3-oxo-acyl-ACP substrates and use them for synthesis of the corresponding shorter chain 3-oxo-AHLs. This explains why in the absence of NADPH only minute amounts of 3oxo- $\mathrm{C}_{12}$-HSL were synthesized in the in vitro reactions, while the levels of 3-oxo- $\mathrm{C}_{8}$ - $\mathrm{HSL}$ and 3 -oxo- $\mathrm{C}_{10}$ - $\mathrm{HSL}$ were greatly elevated (Fig. 4). Consistent with these observations and conclusions, a fabG(Ts) mutant produced overall elevated levels of 3-oxo-AHLs, especially when it was grown at increasing temperatures (Fig. 5; Table 2), presumably since the respective 3-oxo-acylACPs become available for LasI as the growth rate and therefore the demand for fatty acids for other biosynthetic processes decreases. The potential physiological relevance of 3-oxo-AHLs in $P$. aeruginosa other than 3oxo- $\mathrm{C}_{12}-\mathrm{HSL}$, and the regulation of their relative abundances during cellular growth by modulation of FabG activity is currently unclear and awaits further investigation. FabG activity may be controlled at the genetic level (e.g. via transcriptional regulation of $f a b G$ ) or at the protein level (via substrate allosteric effects).

\section{ACKNOWLEDGEMENTS}

This work was supported by NIH grant GM56685 to H. P. Schweizer. We thank Jianzhong Huang at Glaxo SmithKline for the $f a b G(\mathrm{Ts})$ strain and its derivatives, Steven Farrand for the gift of Agrobacterium strains, Matt Parsek for HSL reporter strains and Barbara Iglewski for synthetic 3-oxo- $\mathrm{C}_{12^{-}}$ HSL.

\section{REFERENCES}

Chang, A. C. Y. \& Cohen, S. N. (1978). Construction and characterization of amplifiable multicopy DNA cloning vehicles derived from the P15A cryptic miniplasmid. J Bacteriol 134, 1141-1156.

Chen, H., Cheng, H. \& Bjerkness, M. (1993). One-step Coomassie Brilliant Blue R-250 staining of proteins in polyacrylamide gel. Anal Biochem 212, 295-296.

Cronan, J. E. \& Rock, C. O. (1996). Biosynthesis of membrane lipids. In Escherichia coli and Salmonella, pp. 612-636. Edited by F. C. Neidhardt and others. Washington, DC: American Society for Microbiology Press.

Davies, D. G., Parsek, M. R., Pearson, J. P., Iglewski, B. H., Costerton, J. W. \& Greenberg, E. P. (1998). The involvement of cell-to-cell signals in the development of a bacterial biofilm. Science 280, 295-298.

Heath, R. J. \& Rock, C. O. (1996a). Roles of the FabA and FabZ $\beta$ hydroxyacyl-acyl carrier protein dehydratase in Escherichia coli fatty acid biosynthesis. J Biol Chem 271, 27795-27801.

Heath, R. J. \& Rock, C. O. (1996b). Regulation of fatty acid elongation and initiation by acyl-acyl carrier protein in Escherichia coli. J Biol Chem 271, 1833-1836.

Hoang, T. T. \& Schweizer, H. P. (1997). Fatty acid biosynthesis in Pseudomonas aeruginosa: cloning and characterization of the fab $A B$ operon encoding $\beta$-hydroxydecanoyl-acyl carrier protein dehydratase (FabA) and $\beta$-ketoacyl-acyl carrier protein synthase I (FabB). J Bacteriol 179, 5326-5332.

Hoang, T. T. \& Schweizer, H. P. (1999). Characterization of the Pseudomonas aeruginosa enoyl-acyl carrier protein reductase: a target for triclosan and its role in acylated homoserine lactone synthesis. J Bacteriol 181, 5489-5497.

Hoang, T. T., Karkhoff-Schweizer, R. R., Kutchma, A. J. \& Schweizer, H. P. (1998). A broad-host-range Flp-FRT recombination system for site-specific excision of chromosomallylocated DNA sequences: application for isolation of unmarked Pseudomonas aeruginosa mutants. Gene 212, 77-86.

Hoang, T. T., Ma, Y., Stern, R. J., McNeil, M. R. \& Schweizer, H. P. (1999). Construction and use of low-copy number T7 expression vectors for purification of problem proteins: purification of Mycobacterium tuberculosis RmlD and Pseudomonas aeruginosa LasI and RhlI proteins, and functional analysis of RhlI. Gene 237, 361-371.

Holden, M. T. G., Chhabra, S. R., de Nys, R. \& 14 other authors 
(1999). Quorum-sensing cross talk: isolation and chemical characterization of cyclic dipeptides from Pseudomonas aeruginosa and other Gram-negative bacteria. Mol Microbiol 33, 1254-1266.

Kutchma, A. J., Hoang, T. T. \& Schweizer, H. P. (1999). Characterization of a Pseudomonas aeruginosa fatty acid biosynthetic gene cluster: purification of acyl carrier protein (ACP) and malonyl-coenzyme A:ACP transacylase (FabD). J Bacteriol 181, 5498-5504.

Lambalot, R. H. \& Walsh, C. T. (1995). Cloning, overproduction, and characterization of the Escherichia coli holo-acyl carrier protein synthase. J Biol Chem 270, 24658-24661.

Liss, L. (1987). New M13 host: $\mathrm{DH} 5 \alpha \mathrm{F}^{\prime}$ competent cells. Focus 9 , 13.

Makowski, G. S. \& Ramsby, M. L. (1993). pH modification to enhance the molecular sieving properties of sodium dodecyl sulfate-10\% polyacrylamide gel. Anal Biochem 212, 283-285.

Miller, J. H. (1992). A Short Course in Bacterial Genetics. Cold Spring Harbor, NY: Cold Spring Harbor Laboratory Press.

Moré, M. I., Finger, D., Stryker, J. L., Fuqua, C., Eberhard, A. \& Winans, S. C. (1996). Enzymatic synthesis of a quorum-sensing autoinducer through use of defined substrates. Science 272, 1655-1658.

Parsek, M. R., Val, D. L., Hanzelka, B. L., Cronan, J. E. \& Greenberg, E. P. (1999). Acyl homoserine-lactone quorum-sensing signal generation. Proc Natl Acad Sci U S A 98, 4360-4365.

Passador, L., Cook, J. M., Gambello, M. J., Rust, L. \& Iglewski, B. H. (1993). Expression of Pseudomonas aeruginosa virulence genes requires cell-to-cell communication. Science 260, 1127-1130.

Pesci, E. C., Milbank, J. B. J., Pearson, J. P., McKnight, S., Kende, A. S., Greenberg, E. P. \& Iglewski, B. H. (1999). Quinolone signalling in the cell-to-cell communication system of Pseudomonas aeruginosa. Proc Natl Acad Sci U S A 96, 11229-11234.

Sambrook, J. \& Russell, D. W. (2001). Molecular Cloning, 3rd edn. Cold Spring Harbor, NY : Cold Spring Harbor Laboratory Press.

Schaefer, A. L., Hanzelka, B. L., Parsek, M. R. \& Greenberg, E. P. (2000). Detection, purification, and structural elucidation of the acylhomoserine lactone inducer of Vibrio fischeri luminescence and other related molecules. Methods Enzymol 305, 288-301.

Schweizer, H. P. (1994). Coordinate derepression of alkaline phosphatase, and binding protein dependent transport systems for phosphate and sn-glycerol-3-phosphate by phosphate starvation in Erwinia carotovora subsp. carotovora. Can J Microbiol 40, 310-313.

Schweizer, H. P., Klassen, T. R. \& Hoang, T. (1996). Improved methods for gene analysis and expression in Pseudomonas. In Molecular biology of Pseudomonads, pp. 229-237. Edited by T. Nakazawa, K. Furukawa, D. Haas \& S. Silver. Washington, DC: American Society for Microbiology Press.

Shaw, P. D., Ping, G., Daly, S. L., Cha, C., Cronan, J. E., Rinehart, K. L. \& Farrand, S. K. (1997). Detecting and characterizing $N$-acylhomoserine lactone signal molecules by thin-layer chromatography. Proc Natl Acad Sci U S A 94, 6036-6041.

Singh, P. K., Schaefer, A. L., Parsek, M. R., Moninger, T. O., Welsh, M. J. \& Greenberg, E. P. (2000). Quorum-sensing signals indicate that cystic fibrosis lungs are infected with bacterial biofilms. Nature 407, 762-764.

Val, D. L. \& Cronan, J. E. (1998). In vivo evidence that $S$ adenosylmethionine and fatty acid intermediates are the substrates for the LuxI family of autoinducer synthases. J Bacteriol 180, 2644-2651.

Van Delden, C. \& Iglewski, B. H. (1998). Cell-to-cell signaling and Pseudomonas aeruginosa infections. Emerg Infect Dis 4, 551-560.

Watson, J. M. \& Holloway, B. W. (1978). Chromosome mapping in Pseudomonas aeruginosa. J Bacteriol 133, 1113-1125.

Watson, W. T., Minogue, T. D., Val, D. L., Beck Von Bodman, S. \& Churchill, M. E. A. (2002). Structural basis and specificity of acylhomoserine lactone signal production in bacterial quorum sensing. Mol Cell 9, 685-694.

Received 17 July 2002; revised 4 September 2002; accepted 11 September 2002. 